\title{
Intermolecular Alignment Dependence of Ethylene Glycol Flow on the Chemical Nature of the Solid Surface (Borosilicate and $\mathrm{SnO}_{2}$ )
}

\author{
Cristina M. Quintella ${ }^{a^{*}}$, Cristiane C. Gonçalves ${ }^{a}$, Iuri Pepe ${ }^{b}$, Angelo M. V. Lima ${ }^{a}$ and Ana P. S. Musse ${ }^{a}$ \\ anstituto de Química, Universidade Federal da Bahia, Campus de Ondina, 40170-290, Salvador - BA, Brazil \\ ${ }^{\mathrm{b}}$ Instituto de Física, Universidade Federal da Bahia, Campus de Ondina, 40210-340, Salvador - BA, Brazil
}

\begin{abstract}
Este trabalho estuda o fluxo de etileno glicol (MEG) sobre duas superfícies sólidas com constituições químicas diferentes: borossilicato e dióxido de estanho $\left(\mathrm{SnO}_{2}\right)$. O alinhamento intermolecular, determinado como polarização e anisotropia, mostrou dependência da natureza química do sólido. A razão entre as tensões interfaciais dinâmicas foi $1.09 \pm 0.07$, devendo ser maior para MEG/borossilicato do que para $\mathrm{MEG} / \mathrm{SnO}_{2}$. A razão da capilaridade foi $0.92 \pm 0.06$, sendo menor para MEG/borossilicato. Medidas estáticas do ângulo de contato mostraram valores menores para borossilicato do que para $\mathrm{SnO}_{2}$. Os mapas de polarização e anisotropia apresentaram valores mais altos para $\mathrm{MEG} / \mathrm{SnO}_{2}$, o que pode ser explicado pela alta tensão interfacial do $\mathrm{MEG} /$ borossilicato. Estes resultados são compatíveis com interações de volume mais fortes em $\mathrm{MEG} / \mathrm{SnO}_{2}$ e com contribuições de superfície mais fortes em MEG/borossilicato. Isto pode ser atribuído ao borossilicato ser mais eletronegativo, favorecendo mais as pontes de hidrogênio com o MEG.
\end{abstract}

This work studied ethylene glycol (MEG) flowing on two different solid surfaces, borosilicate and thin dioxide $\left(\mathrm{SnO}_{2}\right)$. Intermolecular alignment, determined as polarization and anisotropy, showed dependence on the solid chemical nature. The ratio between dynamic surface tensions was found $1.09 \pm 0.07$, being stronger for MEG/borosilicate than for MEG/SnO ${ }_{2}$. The capillary ratio found was $0.92 \pm 0.06$, being smaller for MEG/borosilicate. Static contact angle measurements gave lower values for borosilicate than for $\mathrm{SnO}_{2}$. Both polarization and anisotropy maps presented higher values for MEG/SnO , which can be explained by MEG/borosilicate higher interfacial interaction. The results obtained are compatible with stronger bulk phenomena for $\mathrm{MEG} / \mathrm{SnO} \mathrm{O}_{2}$ and with stronger interfacial phenomena for MEG/borosilicate. This may be due to borosilicate being more electronegative, yielding more efficiently hydrogen bonds with MEG.

Keywords: liquid-solid interface, fluorescence depolarization, anisotropy, liquid flow, dynamic surface tension

\section{Introduction}

Most of the liquid-solid interface studies are phenomenological and based on empirical and semi-empirical methods, due to their complexity, and aim mainly to determine interfacial tension ${ }^{1}$. The interfacial tension can be seen as a combined result of the surface chemical nature, its roughness and structures. Several thermodynamical bulk measurements have been used, such as solute activity ${ }^{2}$, heat of immersion determined by enthalpy of immersion ${ }^{3}$ and adsorption isotherms ${ }^{4}$ as a function of relative pressure. Contact angle determination $^{1,5}$ is a visual method widely used, where the interaction is evaluated by the angle of the drop gas-liquid

\footnotetext{
*e-mail: cristina@ufba.br
}

line. This method needs optical magnification of the solidliquid-gas contact line, only achieved with equipment like a magnifying lens system assembled to a goniometer or a high resolution digital image acquisition system.

Contact angles possess hysteresis 6 relative to forward and backward movements that can be quite large, as much as 50 degrees for water on mineral surfaces ${ }^{7}$. This phenomenon has been discussed in terms of transitions between molecular domains ${ }^{8}$, and is usually attributed to several effects like surface roughness, impurities, solvent rearrangements, surface irregularities and structures, etc. It was found ${ }^{9}$ that the minimum patch size necessary to produce hysteresis was approximately $1 \mu \mathrm{m}$. For a flow on a surface with vertical defects, the healing length from a defect to the unperturbed contact line varies linearly with defect width up to a saturation value when the defect was 
approximately $0.75 \mathrm{~mm}$ in width ${ }^{10}$. The contact angle obtained for static interfaces is not reproduced for dynamical phenomena. Some authors studied dynamic contact angles ${ }^{11}$ using macroscopic hydrodynamics analysis where the liquid flows on the solid. They found that the characteristic dimensionless parameter is the capillary number $\mathrm{Ca}=\nu \mu / \gamma$ where $v$ is the flow velocity, $\mu$ is the fluid viscosity and $\gamma$ the interfacial tension. The literature $^{12}$ also concludes that hydrodynamic effects predominate at low contact angles and velocities whilst molecular effects dominate for large contact angles and high velocities.

Most of these studies look at macroscopic properties. Despite the molecular level interaction determination of reaction yields and chemical analysis performances, almost no experimental information is available concerning molecular causes, particularly regarding intermolecular alignment behavior.

Molecular Hydrodynamics ${ }^{13}$ is a recent research branch that studies hydrodynamic processes on a molecular level, i.e., looks at molecular properties, like intermolecular alignment, that determine velocity distributions, reaction yields, equipment performance, and so on.

To our knowledge there are no experimental works in the literature discussing both the interaction that causes interfacial tension and the intermolecular alignment (anisotropy and polarization) within the liquid flow. For the first time we report the flow intermolecular alignment dependence of the solid surface chemical nature.

Fluorescence depolarization is a well established technique that uses polarized light induced fluorescence (PLF) to study intermolecular alignment processes. It has been used in the investigation of biological structures with self-alignment processes like membranes, rotational diffusion of compounds, cell activity, Langmuir-Bloget films, liquid/liquid interfaces ${ }^{14}$, polymer melts ${ }^{15}$, sol-gel matrices ${ }^{16}$, gas flows, etc.

This technique also has been used to study liquid thin free jets. Kenyon et al. ${ }^{17}$ showed that the first lobe central part of the jet has a non-homogeneous pattern of intermolecular alignment and studied its velocity distribution. Quintella et al. ${ }^{18}$ obtained polarization maps of the first four perpendicular lobes of both frontal and lateral views, for several thin free liquid jets, reporting and explaining their lobed structure. Bain et al. ${ }^{19}$ used polarized single-photon counting to study depolarization kinetics with picosecond resolution.

For the fluorescent molecule to be an intermolecular alignment probe, its rotation period (rotational diffusion time) must be approximately equal to its excitation lifetime. Otherwise, the probe would be either nearly rotationally frozen, during its excited lifetime, or would have high rotational diffusion as in very low viscosity liquids. It is also desirable that the probe dipole moments of fundamental and excited states have similar magnitudes as well as orientations parallel to the longitudinal direction of the molecule.

The fluorescent probe absorption of linearly vertical polarized laser is proportional to the cosine square of the angle between the molecular dipole and the laser electric field. If the dipole is parallel to the longitudinal molecular axis, mainly nearly vertical molecules will absorb laser radiation.

Within a liquid there are strong intermolecular shear forces due to short-range interactions between molecular electronic clouds. The shear forces generate macroscopic properties like viscosity and surface tension. In PLF the non-spherical fluorescent probe molecules align themselves with the liquid molecules, due to strain caused by shear forces, thus becoming molecular sensors of the liquid intermolecular alignment.

While excited and before their fluorescent decay, probe molecules can rotate or not depending on the shear forces around them, i.e., depending on the intermolecular alignment of the liquid molecules. If they are in a region of high shear, i.e., a highly microlaminar flow region, the liquid molecules will be highly aligned as well as the probes. When they decay, the fluorescent emission will have almost the same polarization of the laser light.

If the shear is small, like in a microturbulent regimen region, the liquid molecules will not have a preferential alignment direction. The fluorescent probes will have their alignment scrambled, and fluorescence will not have a defined polarization direction. Thus in PLF technique, the fluorescent probe within liquid solutions behaves as a variable birefringent medium, allowing experimental observation of the liquid flow at a molecular level.

Intermolecular alignment can be interpreted either as a bidimensional phenomenon in terms of polarization ${ }^{17}$, or as a three dimensional phenomenon in terms of anisotropy ${ }^{20}$. For linear polarized light excitation, polarization $(\mathrm{P})$ is defined as $\mathrm{P}=\left(\mathrm{I}_{/ /}-\mathrm{I}_{\perp}\right) /\left(\mathrm{I}_{/ /}+\mathrm{I}_{\perp}\right)$ where $\mathrm{I}_{/ /}$and $\mathrm{I}_{\perp}$ are fluorescence components parallel and perpendicular to the laser electric field. Anisotropy ( $\mathrm{r}$ ) is defined as $\mathrm{r}=2 \mathrm{P} /(3-\mathrm{P})=\left(\mathrm{I}_{/ /}-\mathrm{I}_{\perp}\right) /\left(\mathrm{I}_{/ /}+2 \mathrm{I}_{\perp}\right)$.

\section{Experimental}

The experimental setup consists on a PLF laser line (Figure 1). The $514.5 \mathrm{~nm}$ laser beam was generated by a Coherent Inova60 argon. The laser intensity was kept at $50 \mathrm{~mW}$, a safe value as tests showed that saturation 
phenomena started over $80 \mathrm{~mW}$. The laser light, deflected by mirror (E), is focused into the sample, on a $0.02 \mathrm{~mm}^{2}$ diameter spot, by a biconvex borosilicate lens (L1), with $400 \mathrm{~mm}$ focal length. A vertical Glan-Thompson polarizer (P1) guarantees $100 \%$ polarization.

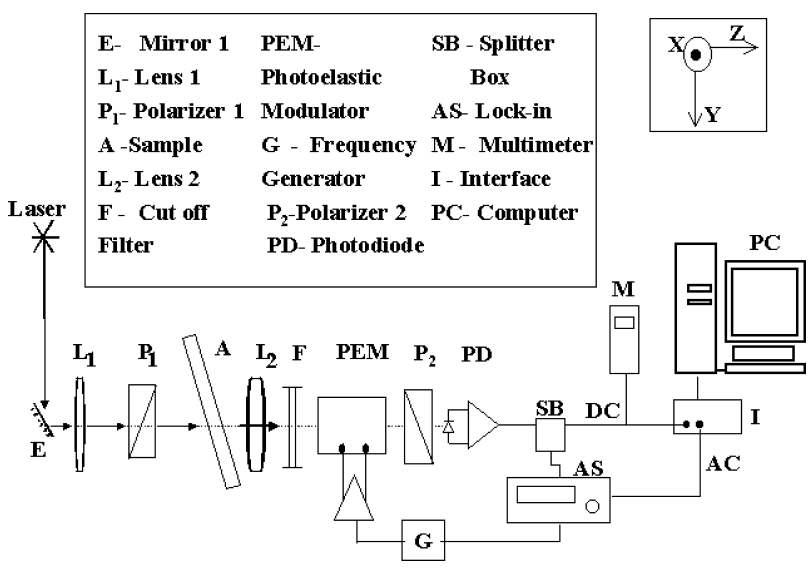

Figure 1. PLF experimental setup.

The fluorescence is collected by a biconvex borosilicate lens (L2), with $50.8 \mathrm{~mm}$ focal length, then passes through a $550 \mathrm{~nm}$ cut-off filter $(\mathrm{F})$ to block the laser radiation. The pure fluorescence light enters the photoelastic modulator (PEM) Conoptics 350-50, this device is driven at $600 \mathrm{~Hz}$ by a frequency generator (G). The PEM modulated output light beam is selected by the horizontal Glan-Thompson polarizer (P2) and reaches the photodiode (PD) at a solid angle of 0.02 sr. This homemade detector consists of an OPT 202 Burr-Brown with $5.22 \mathrm{~mm}^{2}$ active area photodiode, operating as a current to voltage converter with four optional sensibility ranges. The light detector output goes into a splitter box (SB), consisting of a high pass RC filter circuit ( $1 \mathrm{k} \Omega$ and $62 \mathrm{nF}$ ) and a DC coupled output $(1 \mathrm{k} \Omega)$. Here AC and DC components are split and then measured, respectively by an Ithaco NF 3961B lock-in amplifier (AS) and a Tektronix DMM157 digital voltmeter (M). A two channel analog to digital converter (ADC) acquires $\mathrm{AC}$ and $\mathrm{DC}$ voltages through an interface (I) connected to a PC.

Polarization ${ }^{17}(\mathrm{P})$ is defined as $\mathrm{P}=\mathrm{AC} /\left(\mathrm{DC} \times \mathrm{f}_{\mathrm{c}}\right)$ where $\mathrm{f}_{\mathrm{c}}$ is the correction factor due to optical components birefringence, AC and DC are the components of the light detected signal. The correction factor, evaluated by measuring the laser polarization, was 0.20 for both vertical and horizontal polarized light.

A Hitachi VM-E230A video camera with $36 \mathrm{X}$ zoom is connected to a PC to acquire digital images.

The liquid sample position can be varied uniformly in relation to the laser beam direction, using a homemade

two-axis translation frame that is remote controlled by a PC. It is possible to scan flowing systems vertically (Y) and horizontally $(\mathrm{X})$ with $2.5 \mu \mathrm{m}$ resolution in both directions $\left(0.2 \mathrm{~mm}^{2}\right.$ spatial resolution), with repeatability better than $0.1 \%$. The maps here presented were acquired with spatial resolution of $0.7 \mathrm{~mm}$ downstream (Y) and 0.2 mm across stream $(\mathrm{X})$.

A Visual Basic program controls both data acquisition and sample positioning.

The solid surfaces consist of two Perfecta borosilicate microscope slides with $75 \mathrm{~mm}$ x $25 \mathrm{~mm}$ x $0.8 \mathrm{~mm}$. One slide has a $(100 \pm 5) \mathrm{nm}$ layer of $\mathrm{SnO}_{2}$. Both slides were kept for $10 \mathrm{~min}$ in an ultrasound bath with ethanol and dried in a stove at $50{ }^{\circ} \mathrm{C}$ for $15 \mathrm{~min}$ prior the experiment. Both surfaces had roughness below $5 \mathrm{~nm}$, as determined by atomic force microscopy (AFM). The solid surface was fixed at 80 degrees with the horizontal (Figure $2 \mathrm{~b}$ and 3 ). The data here reported was reproduced when using other sets of similar slides.

(a)

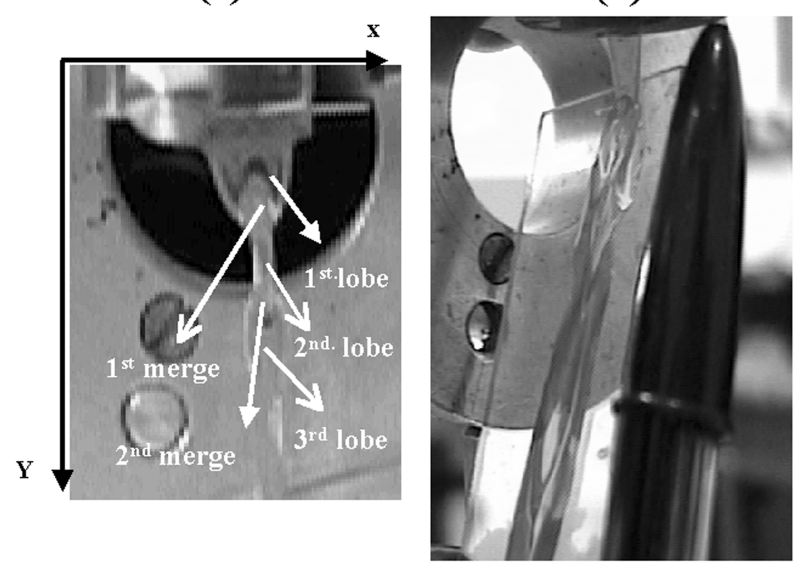

Figure 2. Ethylene glycol flow showing the lobes structure. a) free jet into the air; b) liquid flow on a solid surface.

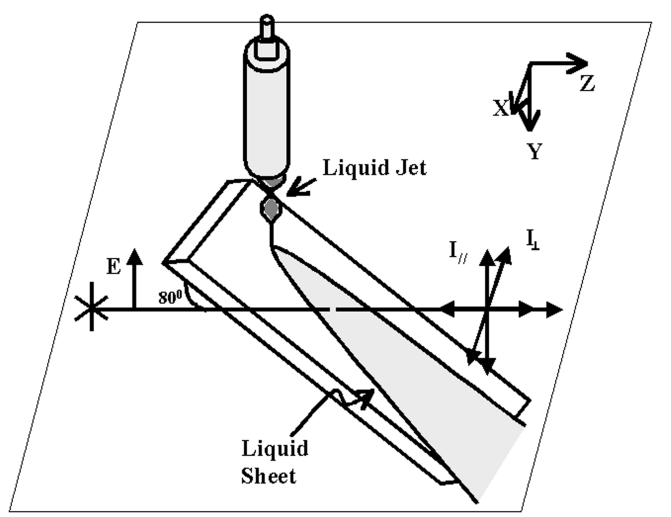

Figure 3. Sample position scheme showing the parabolic liquid film, fluorescence components parallel $\left(\mathrm{I}_{/ /}\right)$and perpendicular $\left(\mathrm{I}_{\perp}\right)$ to the laser electric field. 
The Merck 99.5\% ethylene glycol (MEG), catalog number 10921, passed through a polished sapphire slit nozzle with $100 \mu \mathrm{m} \times 6 \mathrm{~mm}$, generating a thin free jet and impinging the solid surface. The liquid flow was kept at $220 \mathrm{~cm} \mathrm{~s}^{-1}$ constant average velocity, a high value, in order to increase molecular effects as compared to hydrodynamic effects $^{12}$. A homemade thermal bath kept the liquid flow temperature at $15.0 \pm 0.5^{\circ} \mathrm{C}$.

Rhodamine 6G (R6G) (Lambdaphysik, $99.99 \%$ purity) was used as a fluorescent probe. The probe molecule was pumped from $S_{0}$ to $S_{1}$ state ${ }^{21}$ and has dipole moments parallel to the longitudinal direction of the molecule as well as similar intensities ${ }^{22}$. The fluorescence was measured as a function of the R6G concentration. The value of $1.9 \times 10^{-3} \mathrm{~mol} \mathrm{~L}^{-1}$ was chosen in order to maximize the signal, avoiding quenching processes due to excitation migration like Föster transfer ${ }^{23}$. The data here reported was reproduced with lower concentration $\left(0.50 \times 10^{-3} \mathrm{~mol} \mathrm{~L}^{-1}\right)$. The conductivity was obtained with a Multiline P3 pH/LF Conductivity Meter and increased monotonically with R6G concentration from $0.15 \times 10^{-3} \mathrm{~mol} \mathrm{~L}^{-1}$ to $10 \times 10^{-3} \mathrm{~mol} \mathrm{~L}^{-1}$. Conductivity measurements prior and after the experiment gave the same value of $18 \mu \mathrm{S} \mathrm{cm}^{-1}$.

The MEG viscosity decreases with the R6G concentration, being $0.225 \mathrm{~g} \mathrm{~cm}^{-1} \mathrm{~s}^{-1}$ in the experimental conditions.

Under this experimental conditions, the rotational period and excitation lifetime are of the order of $4.2 \mathrm{~ns}$ and 4.9 ns, respectively 24 .

Absorption spectra of pure solid surfaces (liquid flow off) were measured before and after the experiment in order to check any systematic errors due to solid surface adsorbed R6G. No R6G was detected, within the measuring resolution.

Similarly, no fluorescence was detected after turning off the liquid flow, within the experimental sensitivity of light detection.

\section{Results and Discussion}

The evaluation of relative dynamic interfacial tension was obtained using a method developed by our group. A thin free jet (Figure 2a) impinging on the solid surface produces a liquid film (Figures $2 b, 3$ and 4 ). When the jet comes out of the nozzle into the air, it is $100 \mu \mathrm{m}$ thick and consists of a thin sheet in the XY plane, from now on denominated lobe. Then, both lobe borders start to accelerate, due to the air negligible viscosity as compared with the liquid itself and in order to minimize the free surface energy, converging to the jet center. This strong inward movement yields two lateral internal streams. When these streams merge, they generate a liquid sheet (lobe) in the YZ plane. This second lobe is exactly at 90 degrees with respect to the first lobe. Initially its width increases. Then two internal streams start building up at the borders and converge, reducing the lobe width, until they merge, giving rise to the third lobe. The pattern repeats itself, generating up to six lobes. The lobes progressively loose their definition, until the jet becomes rod shaped. (a)

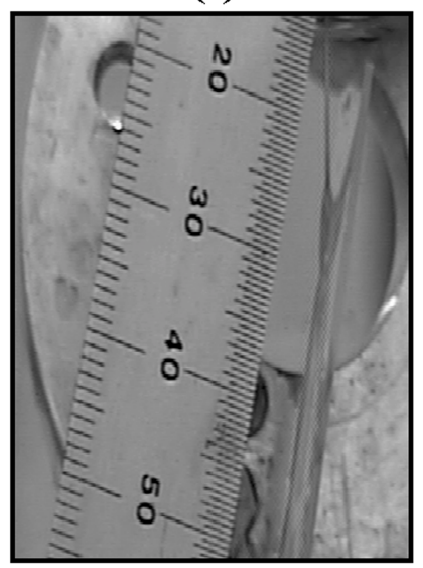

(b)

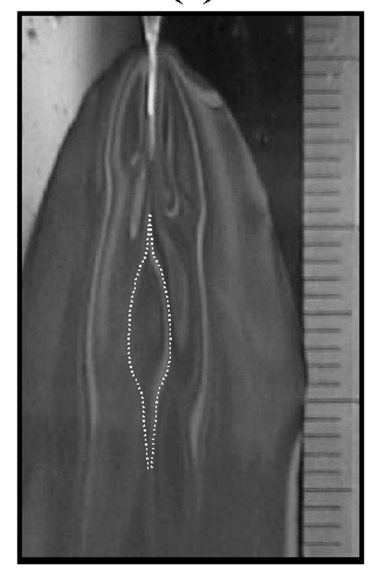

Figure 4. Photo of the ethylene glycol liquid film. a) lateral view with a millimeter scale; b) frontal view.

The first half of the second lobe impinges perpendicularly on the solid surface, yielding a parabolic shaped liquid film on the surface (Figures 3 and $4 \mathrm{~b}$ ). The jet velocity profile is a flattened gaussian when impinging on the surface ${ }^{18}$.

While flowing on the solid surface, the liquid film tends to form a similar lobe pattern. Initially, the liquid film increases its width, generating a middle valley where the flow is the thinnest (Figure 4b). When the width is at maximum, two lateral converging streams are formed. The flow thickness ranged from $150 \mu \mathrm{m}$ at the valley to $1000 \mu \mathrm{m}$ at the borders and was measured with the translation frame, a fixed needle and a magnifying lens.

On the $\mathrm{SnO}_{2}$ surface, the width and length of the MEG lobes are shrunk, when compared with that on borosilicate.

Depending on the interfacial tension, the pattern can have different formation time. The flow vertical movement depends on the gravity, on the stress imposed by the liquid circulation system, and on the interfacial tension. As both flows were at the same average speed, adjusting the imposed shear forces compensates the vertical component of the interfacial tension.

The drag at the interface, due to interfacial tensions, will control the liquid horizontal movements. The valley length was determined with digital images. From the flow average speed, it is possible to infer that it lasted for $(6.0 \pm 0.2) \mathrm{ms}$ on borosilicate and $(5.4 \pm 0.2) \mathrm{ms}$ on $\mathrm{SnO}_{2}$, evidencing lower interface interactions in MEG/borosilicate. These values ratio 
of $(1.09 \pm 0.07)$ gives an idea of the relative dynamic interfacial tensions. The relative capillary ${ }^{11}$ was found to be $(0.92 \pm 0.06)$, being smaller for MEG/borosilicate.

Digital images of $(16 \pm 1) \mu \mathrm{L}$ liquid drops were acquired with a $36 \mathrm{X}$ zoom video camera (vide Experimental section) at laboratory ambient temperature $(23 \pm 1){ }^{\circ} \mathrm{C}$. The images were printed and the contact angles determined being $(19 \pm 2)^{\circ}$ for MEG/borosilicate and $(43 \pm 2)^{\circ}$ for $\mathrm{MEG} / \mathrm{SnO}_{2}$.

The static polarization and anisotropy, measured for optical paths between $300 \mu \mathrm{m}$ and 1,200 $\mu \mathrm{m}$, were $11 \%$ and 0.077 , being constant within the experimental resolution. Below $300 \mu \mathrm{m}$, thermal effects take place, decreasing these values, as expected in a static sample without efficient thermal relaxation processes. In the dynamic system these effects are not seen, as the sample is continuously replaced.

Figures 5 and 6 show the polarization and anisotropy maps, respectively.

(a)

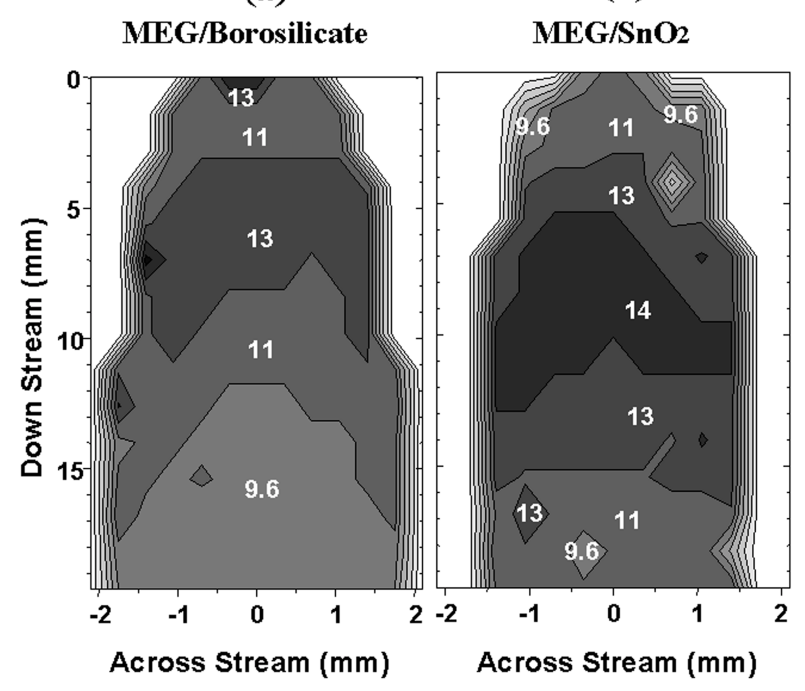

Figure 5. Polarization maps. a) ethylene glycol flowing on borosilicate; b) ethylene glycol flowing on $\mathrm{SnO}_{2}$.

Polarization and anisotropy for the flow are initially higher than static values, which is expected due to the anisotropy imposed by the flow. As the liquid solid interaction takes place, the values tend to decrease. Polarization and anisotropy have their highest values at the valley.

The higher interfacial tension MEG/borosilicate interface has lower polarization.

Considering that the surface roughness is lower than 5 $\mathrm{nm}$ for both solid surfaces, that the surface defect saturation is approximately $0.75 \mathrm{~mm}^{10}$ and that the maps were acquired with resolution over $0.7 \mathrm{~mm}$, if there were any surface structures present, the experiment would not be able to detected them. Thus the results can be seen as depending mainly on the surfaces chemical nature. (a)

MEG/Borosilicate

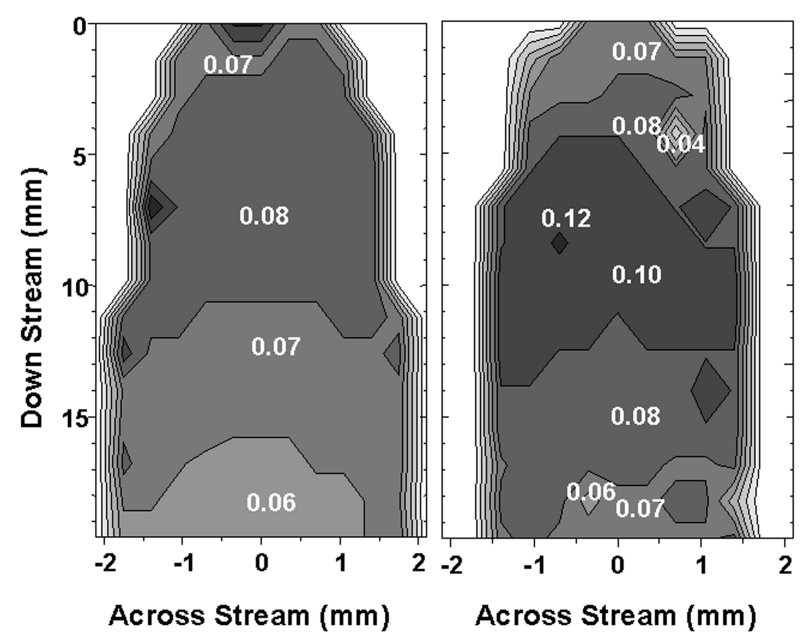

Figure 6. Anisotropy maps. a) ethylene glycol flowing on borosilicate; b) ethylene glycol flowing on $\mathrm{SnO}_{2}$.

The presence of a solid surface generates a boundary layer with friction, due to viscous forces, determined by the intermolecular interactions, which depend on the solid surface chemical nature. These groups of molecules with similar intermolecular alignment are defined as molecular domain $^{8}$ (Figure 7a). When the interaction is stronger, as in borosilicate, the MEG molecules flowing near the surface tend to interact more with the solid surface, slowing down, and behaving like obstacles. As bulk MEG molecules flow on these, they tend to be oriented in several directions, creating several misaligned molecular domains (Figure 7b, A and B). A microturbulent regimen is established and, as a consequence, the intermolecular alignment is scrambled and both polarization and anisotropy decrease.

When the interaction is weaker, as in $\mathrm{SnO}_{2}$, the drag caused by the interaction at the interface decreases. In this case, the molecular domains have higher alignment (Figure $7 \mathrm{~b}, \mathrm{C}$ and D) and bulk phenomena become predominant. The liquid flow intermolecular alignment increases as well as polarization and anisotropy.

From Figures $5 \mathrm{~b}$ and $6 \mathrm{~b}$ it is possible to observe that for $\mathrm{MEG} / \mathrm{SnO}_{2}$ system the range of anisotropy values is higher than the range of polarization values. This effect can be a consequence of MEG low interaction with the interface and thus, the flow on $\mathrm{SnO}_{2}$ presents higher bulk contribution, preserving the flow imposed direction.

The higher polarization and anisotropy, as well as predominant volume contributions may point to higher entropy for $\mathrm{MEG} / \mathrm{SnO}_{2}$ system.

Within the system studied, there are permanent dipoles in R6G and $\mathrm{MEG}^{25}\left(7.6 \times 10^{-30} \mathrm{C} \mathrm{m}\right)$. The R6G concentration was chosen in order to avoid interactions among R6G 
(a)

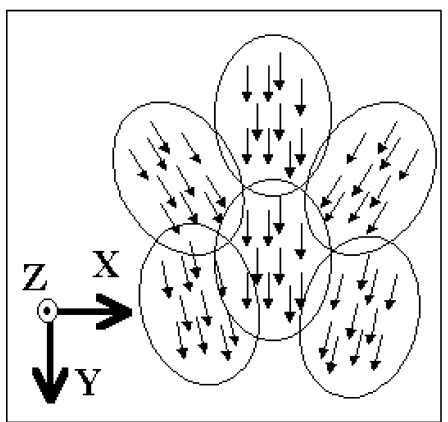

(b)

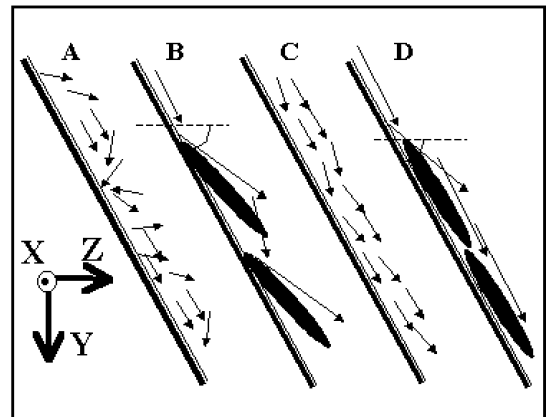

Figure 7. a) molecular domains representation; b) scheme of the flow at the interface showing microturbulence (A, B) and microlaminarity (C, D).

molecules (see Experimental section). The MEG dipoles interact strongly among them and may have a considerable range within the liquid. Their orientation is perturbed by the flow stress due to velocity gradients. The flowing R6G molecules own their deviations from random orientation both to the non spherical molecular geometry and to the induction of MEG solvatation layers (taking in account only steric effects, each R6G can have in the first layer up to 20 MEG molecules).

All the experimental results (static contact angle, liquid film width, valley length, polarization and anisotropy) lead to stronger intermolecular interactions for MEG/borosilicate than for $\mathrm{MEG} / \mathrm{SnO}_{2}$. The interface interactions may be due to hydrogen bonds and dispersion forces (van der Waals).

Considering that MEG has hydroxyls and that both surfaces not only have oxygen atoms, but also have been cationized by ethanol, it is likely that hydrogen bonds may have the highest contribution both for enthalpy and entropy, depending on their nature and density. The borosilicate surface has more electronegative atoms than $\mathrm{SnO}_{2}$ and it is likely that these hydrogen bonds are easily established with borosilicate than with $\mathrm{SnO}_{2}$. Thus the liquid molecules will slip easier on $\mathrm{SnO}_{2}$ than on borosilicate where they will tend to stick. Dispersion forces, when compared with hydrogen bonds, are quite weak, thus their contribution should by low.

Theoretical treatments and numerical simulations are needed to verify these interpretations.

\section{Conclusions}

The interfacial interaction of liquid MEG flowing on borosilicate was found higher than on $\mathrm{SnO}_{2}$, this fact is in agreement with the values of static contact angles.

Both polarization and anisotropy intensities increase for $\mathrm{MEG} / \mathrm{SnO}_{2}$, showing higher bulk contributions due to lower interfacial interaction. When flowing on borosilicate, the interaction increases due to the surface chemical nature, yielding stronger interfacial contributions.
The MEG/ $\mathrm{SnO}_{2}$ anisotropy increases with the flow at higher rate as compared with polarization, pointing to the presence of preferential orientation molecular domains near the MEG/ borosilicate interface.

This strong MEG/borosilicate interaction may be due mainly to stronger hydrogen bonds.

\section{Acknowledgements}

Grants from the Conselho Nacional de Desenvolvimento Científico e Tecnológico (CNPq, Brazil) and PADCT3 supported this work. CTI (Prof. Alaíde P. Mammana) is acknowledged for providing the $\mathrm{SnO}_{2}$ surfaces, IF/USP (Ângela M.P. Passaro) for the atomic force microscopy roughness evaluation and A. J. McCaffery for the sapphire slit nozzle. AMVL and APSM acknowledge their undergraduate research fellowships from PIBICCNPq and CNPq. CMQ acknowledges a senior research scholarship from CNPq.

\section{Electronic supplementary information}

Contains color versions of Figures 2 and 4, with a more detailed description. Available at http://www.sbq.org.br/jbcs

\section{References}

1. Adamson, A. W.; Gast, A. P. Physical Chemistry of Surfaces; John Willey \& Sons, Inc.; New York, 1997.

2. Van Zeggeren, F.; Benson, G. C. Can. J. Chem. 1957, 35,1150 .

3. Zettlemoyer, A. C. Ind. Eng. Chem. Res. 1965, 57, 27.

4. Bilinski, B.; Chibowski, E. Powder Technol. 1983, $35,39$.

5. Johnson, R. E.; Dettre, R. H. J. Phys. Chem. 1964, 68, 1744. 
6. Dettre, R. H.; Johnson, R. E. J. Phys. Chem. 1965 , 69, 1507.

7. Dussan, V. E. B. J. Fluid Mech. 1987, 174, 381.

8. Juzeliunas, G. J. Lumin. 1990, 46, 201.

9. Gaydos, J.; Neumann, A. M. Adv. Colloid Interface Sci. 1994, 49, 197.

10. Marsh, J. A.; Cazabat, A. M. Europhys. Lett. 1993, 23, 45.

11. Ramé, E.; Garoff, S. J. Colloid Interface Sci. 1996, 177, 234.

12. Brochard-Wyart, F.; de Gennes, P. G. Adv. Colloid Interface Sci. 1992, 39,1.

13. Probstein, R. F. Physicochemical Hydrodynamics; Wiley Interscience; New York, 1994.

14. Ishizaka, S.; Nakatani, K.; Habuchi, S.; Kitamura, N. Anal. Chem. 1999, 71, 419.

15. Bur, A. J.; Roth, S. C.; Thomas, C. L. Rev. Sci. Instrum. 2000, 71, 1516.

16. Hungerford, G.; Suhling, K.; Ferreira, J. A. J. Photochem.
Photobiol. A 1999, 129, 71.

17. (a) Kenyon, A. J.; McCaffery, A. J.; Quintella, C. M. Mol. Phys. 1991, 72, 965; (b) Kenyon, A. J.; McCaffery, A. J.; Quintella, C. M.; Winkel, J. F. Mol. Phys. 1993, 74,817 .

18. Quintella, C. M.; McCaffery, A. J. Exp. Fluids. 2001, submitted for publication.

19. Bain, A. J.; Chandna, P.; Butcher, G. Chem. Phys. Lett. 1996, 260, 441.

20. Kwaski, A. Crit. Rev. Anal. Chem. 1993, 23, 459.

21. Aristov, A. V.; Shevandin, V. S. Opt. Spectrosc. 1978, 43, 131.

22. Penzkofer, A.; Wiedmann, J. Opt. Commun. 1980, $35,81$.

23. Knox, R. S. Physica 1968, 39, 361.

24. Scully, A. D.; Matsumoto, A.; Hirayama, S. Chem. Phys. 1991, 157, 253.

25. Lide, D. R. Handbook of Chemistry and Physics; CRC Press; Boca Raton, 1998.

Received: December 20, 2000 Published on the web: September 13, 2001 